\title{
ON STRICTLY CONVEX AND STRICTLY 2-CONVEX 2-NORMED SPACES ॥
}

\author{
C.-S. LIN \\ Department of Mathematics \\ Bishop's University \\ Lennoxville, P.Q. J1M 1Z7, Canada \\ (Received October 2, 1990)
}

\begin{abstract}
In this paper a new duality mapping is defined, and it is our object to show that there is a similarity among these three types of characterizations of a strictly convex 2 -normed space. This enables us to obtain more new results along each of two types of characterizations. We shall also investigate a strictly 2-convex 2 -normed space in terms of the above two different types.
\end{abstract}

KEY WORDS AND PHRASES: Linear 2-normed space, strict convexity, strict 2-convexity, 2-semi-inner product, bounded linear 2-functional, duality mapping.

1991 AMS SUBJECT CLASSIFICATION CODES. 46B05, 46B10, 46C05.

\section{INTRODUCTION.}

This article is a continuation of the paper by Lin [11] where we investigated characterizations of strictly convex and strictly 2-convex 2-normed spaces which were initiated by Diminnie, Gähler and White [5,6]. The concept of strictly convex 2-normed space is 2-dimensional analogue of that of strictly convex normed linear space, an important space in functional analysis, and a strictly 2-convex 2-normed space is its natural generalization. A strictly convex 2-normed space is strictly 2-convex (Theorem 8[6] and Theorem 3 [11]). But the converse is not generally true (Example 2 [6]). Note, however, that strict 2-convexity together with a certain condition is equivalent to strict convexity (Theorem 3 [11]). Most elementary 2-normed spaces originated by Gähler [7] are strictly convex. For example, a 2-normed space of dimension 2, and a 2-inner product space [6]. A strictly convex normed linear space may be characterized in terms of norms by Giles [8], semi-inner products by Berkson [1], or duality mappings by Browder [2], Gudder and Strawther [9] and many others. In this paper a new duality mapping is defined, and it is our object to show that there is a similarity among these three types of characterizations of a strictly convex 2-normed space. This enables us to obtain more new results along each of two types of characterizations. We shall also investigate a strictly 2-convex 2-normed space in terms of the above two different types.

Let $X$ denote a real linear space of dimension greater than one, the following standard definition was introduced in [7]. If $\|.,$.$\| is a real function on X \times X$, then $X$ is called a 2-normed space with a 2-norm $\|.,$. if the following conditions are satisfied:

(i) $\|x, y\|=0$ if and only if $x$ and $y$ are linearly dependent;

(ii) $\|x, y\|=\|y, x\|$;

(iii) $\|a x, y\|=|a|\|x, y\|$ for any real $a$; and 
(iv) $\|x+y, z\| \leq\|x, z\|+\|y, z\|$.

Let $X$ be a 2-normed space throughout this paper. If $x, y, z \in X$ are nonzero vectors, we denote by $V(x), V(x, y)$ and $V(x, y, z)$ the linear manifolds of $X$ generated by $x, x$ and $y, x, y$ and $z$, respectively.

\section{STRICTLY CONVEX 2-NORMED SPACES.}

Recall from [5] that $X$ is said to be strictly convex if $\frac{1}{2}\|x+y, z\|=\|x, z\|-\|y, z\|=1$ for $z \notin V(x, y)$ implies $x=y$. In this section we shall give several characterizations of this space in terms of 2-semi-inner products and duality mappings. But first we need the following lemma which is essential to our consequent theorems, and which is a portion of Theorem 1 in [11] plus three new statements (8), (9), and (10).

LEMMA 1. The following ten statements are equivalent:

(1) $X$ is strictly convex;

(2) $\frac{1}{2}\|x+y, z\|=\|x, z\|-\|y, z\|$ for $z \notin V(x, y)$ implies $x-y$;

(3) $\|x+y, z\|=\|x, z\|+\|y, z\|$ for $z \notin V(x, y)$ implies $x=b y$ for some $b>0$;

(4) $\frac{1}{2}\|x+y, z\|=\|x, z\|-\|y, z\|=0$ for $x \neq y$ implies $z-d(x-y)$ for some $d=0$;

(5) $\|x+a y, z\|=2\|x, z\|$ for $z \notin V(x, y)$ and $a=\|x, z\|\|y, z\|$ implies $x-a y$;

(6) $\|x+y, z\|=\|x, z\|+\|y, z\|$ for $z \notin V(x, y)$ implies $\|y, z\| x=\|x, z\| y$;

(7) $\frac{1}{2}\|x+y, z\|=\|x, z\|-\|y, z\|=0$ for $x \neq y$ implies $\|x, y\|=0$ and $z= \pm\|x, z\|(x-y) /\|x, y\|$;

(8) $\|w+x, z\|=\|w+y, z\| \neq 0$ for all $w \in X$ implies $x=y$;

(9) $\|x-y, z\|=|\|x, z\|-\|y, z\||$ for $z \notin V(x, y)$ implies $x=$ sy for some $s>0$;

(10) $\|x-y, z\|=|\|x, z\|-\|y, z\||$ for $z \notin V(x, y)$ implies $\|y, z\| x=(\|x-y, z\|+\|y, z\|) y$.

PROOF. The equivalence of (1) through (7) was proved in (Theorem $1[11])$, and that (10) $\Rightarrow(9)$ is obvious. That $(9) \Rightarrow(3)$ is clear after we verify the implication $(6) \Rightarrow(10)$.

(6) $\Rightarrow(10)$ : We may write the relation in (10) as $\|x, z\|-\|x-y, z\|+\|y, z\|$. So $\|y, z\|(x-y)=$ $\|x-y, z\| y$ by (6) and the result follows.

(2) $\Rightarrow$ (8): Let $w=x$ and $w=y$ in (8), then $\frac{1}{2}\|x+y, z\|-\|x, z\|-\|y, z\|$ for $z \notin V(x, y)$ implies $x-y$ by (2).

(8) $\Rightarrow$ (2): Suppose that $\frac{1}{2}\|x+y, z\|-\|x, z\|-\|y, z\|$ for $z \notin V(x, y)$ and $x=y$, then $\|w+x, z\|=$ $\|w+y, z\| \neq 0$ for some $w \in X$ (indeed, $w=x$ and $w-y$ ) and $x \neq y$, i.e., (8) does not hold.

The concept of 2-semi-inner product defined by Siddiqui and Rizvi [14] is 2-dimensional analogue of that of the usual semi-inner product in functional analysis. A 2-semi-inner product is a mapping [.,. . .] on $X \times X \times X$ into real numbers such that

(i) $\left[x+x^{\prime}, y \mid z\right]=[x, y \mid z]+\left[x^{\prime}, y \mid z\right]$;

(ii) $[a x, y \mid z]=a[x, y \mid z]$ for any real $a$;

(iii) $[x, x \mid z] \geq 0 ;[x, x \mid z]=0$ if and only if $x$ and $z$ are linearly dependent; and

(iv) $|[x, y \mid z]|^{2} \leq[x, x \mid z][y, y \mid z]$. 
Every 2-normed space can be made into a 2-semi-inner product space, and the norm is given by $\|x, y\|=[x, x \mid y]^{\frac{2}{2}}[14]$.

THEOREM 1. The following nine statements are equivalent:

(1) $X$ is strictly convex (in the sense of Lemma 1);

(2) $[x, y \mid z]=\|x, z\|=\|y, z\|=1$ for $z \notin V(x, y)$ implies $x=y$;

(3) $[x, y \mid z]=\|x, z\|^{2}=\|y, z\|^{2}$ for $z \notin V(x, y)$ implies $x=y$;

(4) $[w, x \mid z]=[w, y \mid z]$ for $z \notin V(x, y, w)$ and all $w \in X$ implies $x=y$;

(5) $[a x, y \mid z]=\|x, z\|^{2}$ for $z \notin V(x, y)$ implies $x=a y$ for some $a>0$, and $a=1$ if $\|x, z\|=\|y, z\|$;

(6) $[x, y \mid z]=\|x, z\|\|y, z\|$ for $z \notin V(x, y)$ implies $x=a y$ for some $a>0$;

(7) $[x, y \mid z]=\|x, z\|^{2}=\|y, z\|^{2} \neq 0$ for $x \neq y$ implies $z=d(x-y)$ for some $d \neq 0$;

(6') $[x, y \mid z]=\|x, z\|\|y, z\|$ for $z \notin V(x, y)$ implies $\|y, z\| x=\|x, z\| y$;

(7') $[x, y \mid z]=\|x, z\|^{2}=\|y, z\|^{2} \neq 0$ for $x \neq y$ implies $\|x, y\| \neq 0$ and $z= \pm\|x, z\|(x-y)\|x, y\|$.

PROOF. The following implications are routine: $(2) \Leftarrow(5) \Leftarrow\left(6^{\prime}\right) \Rightarrow(6) \Rightarrow(3) \Rightarrow(2)$ and $\left(7^{\prime}\right) \Rightarrow(7)$. So let us prove that $(3) \Rightarrow(4) \Rightarrow(1) \Rightarrow\left(6^{\prime}\right),(2) \Rightarrow(1) \Rightarrow\left(7^{\prime}\right)$ and $(7) \Rightarrow(1)$.

$(1) \Rightarrow\left(6^{\prime}\right)$ : Let $[x, y \mid z]=\|x, z\|\|y, z\|$ for $z \notin V(x, y)$, then $(\|x, z\|+\|y, z\|)\|y, z\|-[x+y, y \mid z] \leq$ $\|x+y, z\|\|y, z\| \leq(\|x, z\|+\|y, z\|)\|y, z\|$, or $\|x+y, z\|=\|x, z\|+\|y, z\|$. Hence $\|y, z\| x=\|x, z\| y$ by (6) in Lemma 1.

(3) $\Rightarrow$ (4): Let $w=x$ in (4), then $\|x, z\|^{2}=[x, y \mid z] \leq\|x, z\|\|y, z\|$, or $\|x, z\| \leq\|y, z\|$. If $w=y$, then $\|y, z\| \leq\|x, z\|$ similarly. Hence $\|x, z\|=\|y, z\|$ and $x=y$ by (3).

(4) $\Rightarrow$ (1): Suppose that $X$ is not strictly convex, i.e., $\frac{1}{2}\|x+y, z\|-\|x, z\|=\|y, z\|$ for $z \notin V(x, y)$ and $x \neq y$, we have to show that $[w, x \mid z]=[w, y \mid z]$ for $z \notin V(x, y, w)$ and some $z$ 's implies $x \neq y$. Since $\|x, z\|=\|y, z\|$ by the proof $(3) \Rightarrow(4)$ we have $[x, y \mid z]=\|x, z\|\|y, z\|$. As in the proof $(1) \Rightarrow\left(6^{\prime}\right)$ we conclude that $\frac{1}{2}\|x+y, z\|=\|x, z\|=\|y, z\|$.

(2) $\Rightarrow(1):$ Let $\frac{1}{2}\|x+y, z\|=\|x, z\|=\|y, z\|=1$ and $x \neq y$, then, with the aid of the proof $(1) \Rightarrow\left(6^{\prime}\right)$, we can show easily that $[x, y \mid z]=\|x, z\|=\|y, z\|=1$ implies $x \neq y$.

$(1) \Rightarrow\left(7^{\prime}\right):$ Let $x \neq y$ and $[x, y \mid z]=\|x, z\|^{2}=\|y, z\|^{2}$, so $[x, y \mid z]=\|x, z\|\|y, z\|$, then $\frac{1}{2}\|x+y, z\|=$ $\|x, z\|=\|y, z\|$ by the proof $(1) \Rightarrow\left(6^{\prime}\right)$. Hence $\|x, z\| \neq 0$ and $z= \pm\|x, z\|(x-y) /\|x, y\|$ by (7) in Lemma 1.

(7) $\Rightarrow(1)$ : Suppose by contrapositive that (4) in Lemma 1 does not hold, then by the proof $(1) \Rightarrow\left(6^{\prime}\right)$ it is easily seen that (7) does not hold, and the proof of the theorem is complete.

Motivated by the concepts of bounded linear functionals, and duality mappings on normed linear spaces [2,9], bounded linear 2-functionals on 2-normed spaces were introduced by White [15], and associated duality mappings were defined in [3]. Let $M$ and $N$ be linear manifolds of $X$, a bounded linear 2 -functional is a mapping $f$ on $M \times N$ into real numbers such that

(i) $f\left(x+x^{\prime}, y+y^{\prime}\right)=f(x, y)+f\left(x, y^{\prime}\right)+f\left(x^{\prime}, y\right)+f\left(x^{\prime}, y^{\prime}\right)$;

(ii) $f(a x, b y)=a b f(x, y)$ for any real numbers $a$ and $b$; and

(iii) $|f(x, y)| \leq k\|x, z\|$ for some $k \geq 0$ and all $(x, y) \in M \times N$. 
In this case the norm of $f$ is defined by

$$
\|f\|=\inf \{k:|f(x, y)| \leq k\|x, y\|,(x, y) \in M \times N\}
$$

It can be shown that $|f(x, y)| \leq\|f\|\|x, y\|$ and $f(x, y)=0$ if $x \in V(y)$ [15]. We need also a result which is similar to the Hahn-Banach theorem of functional analysis: If $x, z \in X$ and $x \notin V(z)$, then there exists a bounded linear 2-functional $f$ on $X \times V(z)$ such that $f(x, z)=\|x, z\|$ and $\|f\|=1[6,13,15]$.

The following duality mappings defined in [3] are 2-dimensional analogues of usual duality mappings on a normed linear space $[2,9]$ :

$$
\begin{aligned}
& I(x, z)=\left\{f \in X_{z}^{*}: f(x, z)=\|f\|\|x, z\|\right\} \text { and } \\
& J(x, z)=\left\{f \in X_{z}^{*}: f(x, z)=\|f\|\|x, z\|,\|f\|=\|x, z\|\right\}
\end{aligned}
$$

with duality mappings $I, J: X \times V(z) \rightarrow 2^{X_{z}^{*}}$, where $X_{z}^{*}$ is the space of all bounded linear 2 -functionals on $X \times V(z)$.

Evidently the following assertions are true: (a) $J(x, z) \subseteq I(x, z)$; (b) $I(x, z)=X_{z}^{*}$ if and only if $x \in V(z)$; (c) $I(x, z)=I(c x, d z)=c d I(x, z)$ for $c, d>0$; (d) $0 \neq f \in I(x, z)$ for $x \notin V(z)$ implies $f \in J(c x, z)$ for some $c>0$; and (e) If $x \notin V(z)$, then there exists an $f \in J(x, z)$ with $f=0$ (by the Hahn-Banach theorem stated in above).

Let us define another type of duality mapping as follows:

DEFINITION. Let $I^{\prime}(x, z)$ be the same as $I(x, z)$ which has the following additional properties:

(i) $\|x, z\| \geq\|y, z\|$ if and only if $\|f\| \geq\|g\|$ for $z \notin V(x, y), f \in I(x, z)$ and $g \in I(y, z)$; and

(ii) $\|x, z\| \geq\|x, w\|$ if and only if $\|f\| \geq\|h\|$ for $x \notin V(z, w), f \in I(x, z)$ and $h \in I(x, w)$.

It follows easily from (i) that $f \in I^{\prime}(x, z) \cap I^{\prime}(y, z)$ for $z \notin V(x, y)$ if and only if $f(x, z)=$ $\|f\|\|x, z\|, f(y, z)=\|f\|\|y, z\|$ and $\|x, z\|=\|y, z\|$. A similar result from (ii) is obtainable.

LEMMA 2. If $0 \neq f \in I^{\prime}(x, z), 0 \neq g \in I^{\prime}(y, z)$ for $x \neq y$ and $z \notin V(x, y)$, then

(1) $(f-g)(x-y, z) \geq 0$;

(2) $(f-g)(x-y, z)=0$ if and only if $f(y, z)=\|f\|\|y, z\|, g(x, z)=\|g\|\|x, z\|$ and $\|x, z\|-\|y, z\|$;

(3) $(f-g)(x-y, z)=0$ if and only if $f, g \in I^{\prime}(x, z) \cap I^{\prime}(y, z)$.

PROOF. (1) and (2) are straightforward computations and can be found in ([10] p. 379). Indeed, $(f-g)(x-y, z)-(\|f\|-\|g\|)(\|x, z\|-\|y, z\|)+[\|f\|\|y, z\|-f(y, z)]+[\|g\|\|x, z\|-g(x, z)] \geq 0$. (3) is consequences of $(2)$ and a previous remark.

In a similar manner we can prove the following analogous result.

LEMMA 3. If $0 \neq f \in I^{\prime}(x, z), 0 \neq g \in I^{\prime}(x, w)$ for $z \neq w$ and $x \notin V(z, w)$, then

(1) $(f-g)(x, z-w) \geq 0$;

(2) $(f-g)(x, z-w)=0$ if and only if $f(x, w)=\|f\|\|x, w\|, g(x, z)-\|g\|\|x, z\|$ and $\|x, z\|=\|x, w\|$

(3) $(f-g)(x, z-w)=0$ if and only if $f, g \in I^{\prime}(x, z) \cap I^{\prime}(x, w)$.

Obviously, $I^{\prime}$ in Lemma 2 and 3 may be replaced by $J$. Let \# denote the inclusion relation $\subseteq \supseteq$ or $=$.

THEOREM 2. If $x, y \neq 0$, then the following thirteen statements are equivalent:

(1) $X$ is strictly convex (in the sense of Lemma 1 ); 
(2) $I(x, z) \cap I(y, z) \neq \varnothing$ for $z \notin V(x, y)$ implies $x=a y$ for some $a>0$;

(3) $I(x, z) \# I(y, z)$ for $z \notin V(x, y)$ implies $x=a y$ for some $a>0$;

(4) $J(x, z) \cap J(y, z) \neq \varnothing$ for $z \notin V(x, y)$ implies $x=y$;

(5) $J(x, z) J(y, z)$ for $z \notin V(x, y)$ implies $x=y$;

(6) $I^{\prime}(x, z) \cap I^{\prime}(y, z) \neq \varnothing$ for $z \notin V(x, y)$ implies $x=y$;

(7) $I^{\prime}(x, z) I^{\prime}(y, z)$ for $z \notin V(x, y)$ implies $x=y$;

(8) If $0 \neq f \in I^{\prime}(x, z)$ and $0 \neq g \in I^{\prime}(y, z)$ for $x \neq y$ and $z \notin V(x, y)$, then $(f-g)(x-y, z)>0$;

(9) $J(x, z) \cap J(y, z) \neq \varnothing$ for $x \neq y$ implies $z=d(x-y)$ for some $d \neq 0$;

(2') $I(x, z) \cap I(y, z) \neq \varnothing$ for $z \notin V(x, y)$ implies $\|y, z\| x=\|x, z\| y$;

(3') $I(x, z) \# I(y, z)$ for $z \notin V(x, y)$ implies $\|y, z\| x=\|x, z\| y$;

(8) If $0 \neq f \in J(x, z)$ and $0 \neq g \in J(y, z)$ for $x \neq y$ and $z \notin V(x, y)$, then $(f-g)(x-y, z)>0$;

$\left(9^{\prime}\right) J(x, z) \cap J(y, z) \neq \varnothing$ for $x \neq y$ implies $\|x, y\| \neq 0$ and $z= \pm\|x, z\|(x-y) /\|x, y\|$.

PROOF. The proof of $\left(2^{\prime}\right) \Rightarrow(2) \Rightarrow(3),\left(2^{\prime}\right) \Rightarrow\left(3^{\prime}\right) \Rightarrow(3)$ and $\left(9^{\prime}\right) \Rightarrow(9)$ are trivial. Equivalences of (1), (4), (5), (6) and (7) are clear after we verify the implications $(3) \Rightarrow(1) \Rightarrow\left(2^{\prime}\right)$. ( $\left.8^{\prime}\right)$ is, of course, a special case of (8).

$(1) \Rightarrow\left(2^{\prime}\right):$ Let $0 \neq f \in I(x, z) \cap I(y, z)=I(x, z) \cap I(\|x, z\| y /\|y, z\|, z)$, then $\|f\| \| x+(\|x, z\| y /\|y, z\|)$, $z\|\geq f(x+(\|x, z\| y /\|y, z\|), z)=2\| f\|\| x, z\|\geq\| f\|\| x+(\|x, z\| y /\|y, z\|), z \|$, or $\|x+(\|x, z\| y\|y, z\|), z\|=$ $2\|x, z\|$ and hence $\|y, z\| x=\|x, z\| y$ by (5) in Lemma 1 .

(3) $\Rightarrow(1)$ : Without loss of generality we may assume that $0 \neq f \in I(x, z) \subseteq I(y, z)$ in (3). Suppose that $\|x+y, z\|=\|x, z\|+\|y, z\|$ and $x \neq b y$ for all $b>0$, i.e., the negation of (3) in Lemma 1 , we have to show that $f \in I(x, z) \subseteq I(y, z)$ implies $x \neq b y$ for all $b>0$. This follows from the relation $\|f\|\|x+y, z\| \geq f(x+y, z)=\|f\|(\|x, z\|+\|y, z\|) \geq\|f\|\|x+y, z\|$, or $\|x+y, z\|=\|x, z\|+\|y, z\|$.

(6) $\Rightarrow(8)$ : Let $0 \neq f \in I^{\prime}(x, z), 0 \neq g \in I^{\prime}(y, z), x \neq y, z \notin V(x, y)$ and $(f-g)(x-y, z)=0$, then $f \in I^{\prime}(x, z) \cap I^{\prime}(y, z)$ by Lemma 2 , and $x \neq y$. Thus (6) does not hold.

(8) $\Rightarrow(6)$ : If $f \in I^{\prime}(x, z) \cap I^{\prime}(y, z)$ and if $x \neq y$, then $0=(f-f)(x-y, z)>0$ by (8) yielding a contradiction.

$(1) \Rightarrow\left(9^{\prime}\right)$ : For $x \neq y$ let $0 \neq f \in J(x, z) \cap J(y, z)$, then $\|x, z\|-\|y, z\|-\|f\| \neq 0$. It follows easily that $\frac{1}{2}\|x+y, z\|=\|x, z\|=\|y, z\| \neq 0$. Hence $\|x, y\| \neq 0$ and $z= \pm\|x, z\|(x-y) /\|x, y\|$ by (7) in Lemma 1 .

(9) $\Rightarrow$ (1): Consider the negation of (4) in Lemma 1, i.e., $\frac{1}{2}\|x+y, z\|-\|x, z\|=\|y, z\| \neq 0, x \neq y$ and $z \neq d(x-y)$ for all $d \neq 0$, then as in the proof $(1) \Rightarrow\left(9^{\prime}\right)$ we can easily conclude that (9) does not hold.

REMARKS. (a) That $J(x, z) \cap J(y, z) \neq \varnothing$ in (9) and (9') above may be replaced, of course, by $J(x, z) \# J(y, z)$ without any other change in the statements; (b) $J$ in (9) and $\left(9^{\prime}\right)$ may be replaced by $I^{\prime}$ if $\|x, z\|$ or $\|y, z\| \neq 0$ in addition to the conditions; (c) Though (2) appeared in ([3] Theorem 1), our proof is direct and much simpler. (4) is in ([3] Corollary 3). (8) was discussed in ([10] Theorem 2.5) with a different type of duality mapping; (d) Note that a duality mapping which satisfies the statement (8) is said to be strictly monotone [10] (cf. [2,9]). In other words, $X$ is strictly convex if and only if $I^{\prime}$ or $J$ is strictly monotone. 


\section{STRICTLY 2-CONVEX 2-NORMED SPACES.}

According to [6] $X$ is said to be strictly 2-convex if $\|x+z, y+z\| / 3=\|x, y\|=\|y, z\|-\|z, x\|=1$ implies $z=x+y$. We now turn to the investigation of this space in terms of 2-semi-inner products and duality mappings. To this end we require first the next result which is a portion of Theorem 2 in [11].

LEMMA 4. The following four statements are equivalent:

(1) $X$ is strictly 2-convex;

(2) $\|x+z, y+z\|=\|x, y\|+\|y, z\|+\|z, x\|$ for $\|x, y\|\|y, z\|\|z, x\| \neq 0$ implies $z=b x+c y$ for some $b, c>0$

(3) $\|b x+z, c y+z\|=3\|b x, z\|$ for $\|x, y\|\|y, z\|\|z, x\| \neq 0$ implies $z=b x+c y$, where $b-\|y, z\| /$ $\|x, y\|$ and $c=\|x, z\| /\|x, y\|$.

(4) $\|x+z, y+z\|=\|x, y\|+\|y, z\|+\|z, x\|$ for $\|x, y\|\|y, z\|\|z, x\|=0$ implies $z=b x+c y$, where $b$ and $c$ are as in (3).

In order to be able to prove the next theorem we shall use one of the basic properties of a 2-norm that $\|a x+b y, y\|=|a|\|x, y\|$ for any real numbers $a$ and $b[7]$.

THEOREM 3. The following five statements are equivalent:

(1) $X$ is strictly 2 -convex (in the sense of Lemma 4);

(2) $[-x, y \mid y+z]-(\|x, y\|+\|x, z\|)\|y, z\|$ for $\|x, y\|\|y, z\|\|z, x\| \neq 0$ implies $z=b x+c y$ for some $b, c>0$;

(3) $\frac{1}{2}[-x, y \mid y+z]=\|x, y\|^{2}=\|y, z\|^{2}=\|z, x\|^{2} \neq 0$ implies $z=x+y$;

(4) $\frac{1}{2}[-x, y \mid y+z]=\|x, y\|=\|y, z\|=\|z, x\|=1$ implies $z=x+y$;

(2') $[-x, y \mid y+z]=(\|x, y\|+\|z, x\|)\|y, z\|$ for $\|x, y\|\|y, z\|\|z, x\| \neq 0$ implies $z=b x+c y$, where $b=\|y, z\| /\|x, y\|$ and $c=\|x, z\| /\|x, y\|$.

PROOF. The following implications are trivial: $\left(2^{\prime}\right) \Rightarrow(2) \Rightarrow(3) \Rightarrow(4)$.

$(1) \Rightarrow\left(2^{\prime}\right): \quad$ If $[-x, y \mid y+z]=(\|x, y\|+\|x, z\|)\|y, z\|$, then $(\|x, y\|+\|y, z\|+\|z, x\|)\|y, z\|=$ $[y-x, y \mid y+z] \leq\|y-x, y+z\|\|y, z\|=\|(y+z)-(x+z), y+z\|\|y, z\|=\|x+z, y+z\|\|y, z\| \leq(\|x, y\|+$ $\|y, z\|+\|z, x\|)\|y, z\|$, or $\|x+z, y+z\|=\|x, y\|+\|x, z\|+\|y, z\|$ and the result follows by (4) in Lemma 4.

(4) $\Rightarrow$ (1): If $\|x+z, y+z\| / 3=\|x, y\|=\|y, z\|=\|z, x\|=1$ and $z \neq x+y$, we have to show that $\frac{1}{2}[-x, y \mid y+z]=\|x, y\|=\|y, z\|=\|z, x\|=1$ implies $z \neq x+y$. But this is clear from the proof in above.

THEOREM 4. In the following let $I(u, v), J(u, v)$ and $I^{\prime}(u, v)$ be defined as in the previous section, and let $u \notin V(v)$, then the following seven statements are equivalent:

(1) $X$ is strictly 2-convex (in the sense of Lemma 4);

(2) $I(x, y) \cap I(x, z) \cap I(z, y)=\varnothing$ implies $z=b x+c y$ for some $b, c>0$;

(3) $J(x, y) \cap J(x, z) \cap J(z, y) \neq \varnothing$ implies $z=x+y$;

(4) $I^{\prime}(x, y) \cap I^{\prime}(x, z) \cap I^{\prime}(z, y) \neq \varnothing$ implies $z=x+y$;

(5) If $0 \neq f \in I^{\prime}(x, y), 0 \neq g \in I^{\prime}(x, z)$ and $0 \neq h \in I^{\prime}(z, y)$ for $z \neq x+y$, then $(f-h)(x-z, y)$ and $(f-g)(x, y-z)>0$;

(2') $I(x, y) \cap I(x, z) \cap I(z, y) \neq \varnothing$ implies $z=b x+c y$ for $b=\|y, z\|\|x, y\|$ and $c=\|x, z\|\|x, y\|$; 
(5') If $0 \neq f \in J(x, y), 0 \neq g \in J(x, z)$ and $0 \neq h \in J(z, y)$ for $z \neq x+y$, then $(f-h)(x-z, y)$ and $(f-g)(x, y-z)>0$.

PROOF. That $\left(2^{\prime}\right) \Rightarrow(2)$ is trivial. $\left(5^{\prime}\right)$ is a special case of $(5)$, and it is clear that we need to verify that $(2) \Rightarrow(1) \Rightarrow\left(2^{\prime}\right)$ and $(4) \Leftrightarrow(5)$ only.

$(1) \Rightarrow\left(2^{\prime}\right)$ : Let $0 \neq f \in I(x, y) \cap I(x, z) \cap I(z, y)=I(b x, c y) \cap I(b x, z) \cap I(z, c y)$, where $b=\|y, z\| /$ $\|x, y\|$ and $c=\|x, z\|\|x, y\|$, then $\|f\|\|b x+z, c y+z\| \leq\|f\|(\|b x, c y\|+\|b x, z\|+\|z, c y\|)=f(b x+z$, $c y+z), \leq\|f\|\|b x+z, c y+z\|$, or $\|b x+z, c y+z\|=\|b x, c y\|+\|b x, z\|+\|z, c y\|=3\|b x, z\|$, and $\|x, y\|$ $\|y, z\|\|z, x\| \neq 0$ by assumption. So $z=b x+c y$ by (3) in Lemma 4.

(2) $\Rightarrow(1)$ : Consider the negation of (2) in Lemma 4, i.e., $\|x+z, y+z\|-\|x, y\|+\|x, z\|+\|z, y\|$, $\|x, y\|\|y, z\|\|z, x\| \neq 0$ and $z \neq b x+c y$ for all $b, c>0$, we have to show that $0 \neq f \in I(x, y) \cap$ $I(x, z) \cap I(z, y)$ implies $z \neq b x+c y$ for all $b, c>0$. This follows from the relation $\|f\|\|x+z, y+z\| z$ $f(x+z, y+z)=\|f\|(\|x, y\|+\|x, z\|+\|z, y\|) \geq\|f\|\|x+z, y+z\|$, or $\|x+z, y+z\|=\|x, y\|+\|x, z\|+$ $\|z, y\|$.

(4) $\Rightarrow(5): \quad$ Let $\quad 0 \neq f \in I^{\prime}(x, y), 0 \neq g \in I^{\prime}(x, z), 0 \neq h \in I^{\prime}(z, y),(f-h)(x-z, y)=0=(f-g)$ $(x, y-z)$ and $z \neq x+y$, i.e., the negation of (5), then $f \in I^{\prime}(x, y) \cap I^{\prime}(z, y) \cap I^{\prime}(x, z)$ by Lemma 2 and 3 , and $z \neq x+y$. Thus (4) does not hold.

(5) $\Rightarrow(4)$ : If $f \in I^{\prime}(x, y) \cap I^{\prime}(x, z) \cap I^{\prime}(z, y)$ and suppose that $z \neq x+y$, then $0=(f-f)(x-z, y)>0$ by (5) yielding a contradiction, and the proof of the theorem is complete.

REMARK. (2) in Theorem 4 appeared in ([4] Theorem 1.2) except that the domain of the duality mapping $I$ has been changed. The change is unnecessary.

\section{REFERENCES}

1. BERKSON, E., Some Types of Banach Spaces, Hermitian Operators, and Bade Functionals, Trans. Amer. Math. Soc. 116 (1965), 376-385.

2. BROWDER, F. E., Multi-valued Monotone Nonlinear Mappings and Duality Mappings in Banach Spaces, Trans. Amer. Math. Soc. 118 (1965), 338-351.

3. CHO, Y. J., HA K. S. and KIM, W. S., Strictly Convex Linear 2-Normed Spaces, Math. Japon. 26 (1981), 475-478.

4. CHO, Y. J., PARK, B. H. and PARK, K. S., Strictly 2-Convex Linear 2-Normed Spaces, Math. Japon. 24 (1982), 609-612.

5. DIMINNIE, C., GAHLER, S. and WHITE, A., Strictly Convex Linear 2-Normed Spaces, Math. Nachr. 59 (1974), 319-324.

6. DIMINNIE, C., GAHLER, S. and WHITE, A, Remarks on Strictly Convex and Strictly 2-Convex 2-Normed Spaces, Math. Nachr. 88 (1979), 363-372.

7. GAHLER, S., Linear 2-Normiente Räume, Math. Nachr. 28 (1965), 1-43.

8. GILES, J. R., Class of Semi-Inner-Product Spaces, Trans. Amer. Math. Soc. 129 (1967), 436-446.

9. GUDDER, S. and STRAWTHER, D., Strictly Convex Normed Linear Spaces, Proc. Amer. Math. Soc. 59 (1976), 263-267.

10. HA, K. S., CHO, Y. J. and WHITE, A., Strictly Convex and Strictly 2-Convex 2-Normed Spaces, Math. Japon. $\underline{33}$ (1988), 375-384.

11. LIN, C. -S., On Strictly Convex and Strictly 2-Convex 2-Normed Spaces, Math. Nachr., to appear.

12. LIN, C. -S., On Strictly Convex and Normally Convex Spaces, preprint.

13. MABIZELA, S., On Bounded Linear 2-Functionals, Math. Japon. 35 (1990), 51-55.

14. SIDDIQUI, A. H. and RIZVI, S. M., 2-Semi-Inner Product Spaces I, Math. Japon. 21 (1976), 391-397.

15. WHITE, A., 2-Banach Spaces, Math. Nachr. 42 (1969), 43-60. 


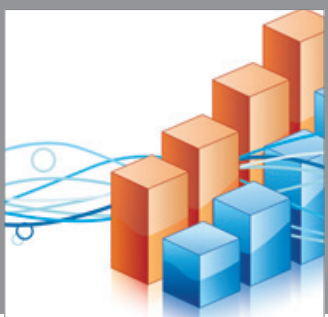

Advances in

Operations Research

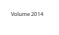

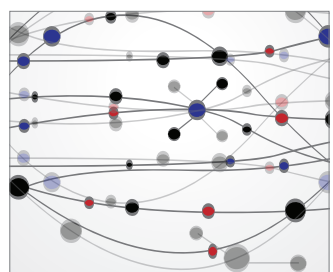

\section{The Scientific} World Journal
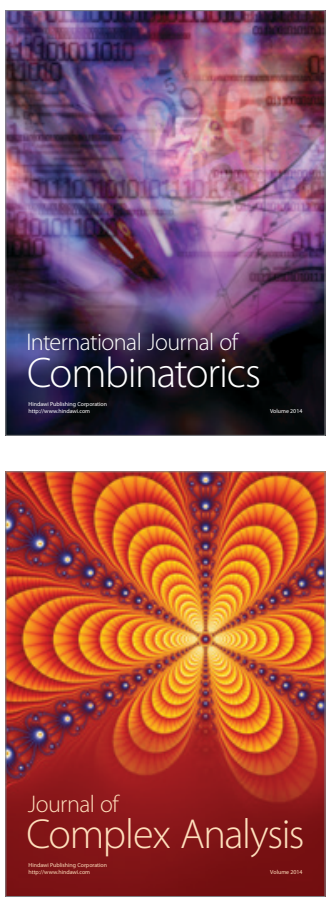

International Journal of

Mathematics and

Mathematical

Sciences
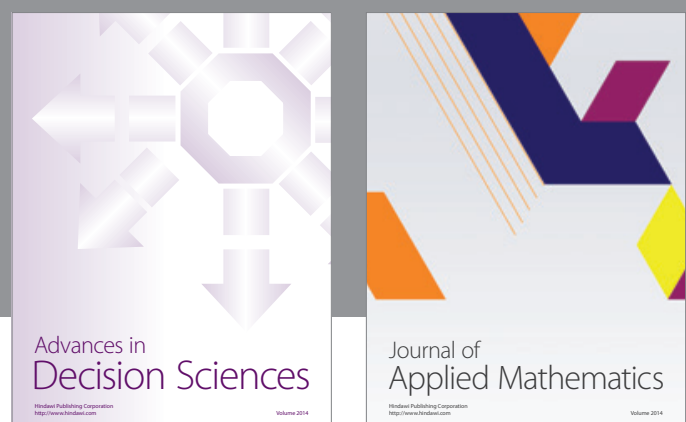

Journal of

Applied Mathematics
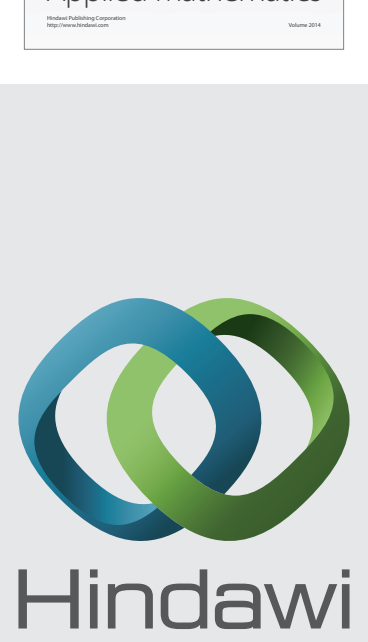

Submit your manuscripts at http://www.hindawi.com
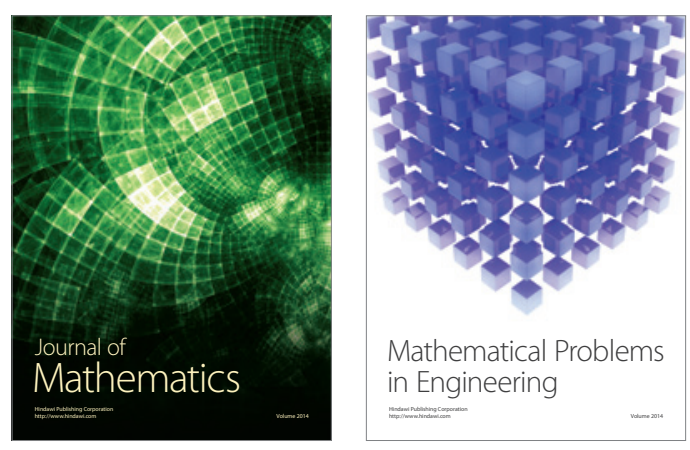

Mathematical Problems in Engineering
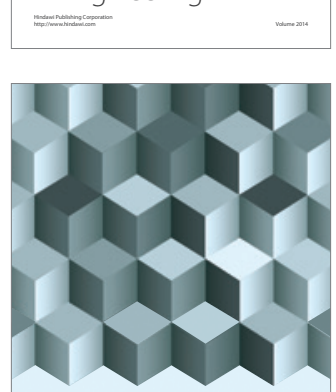

Journal of

Function Spaces
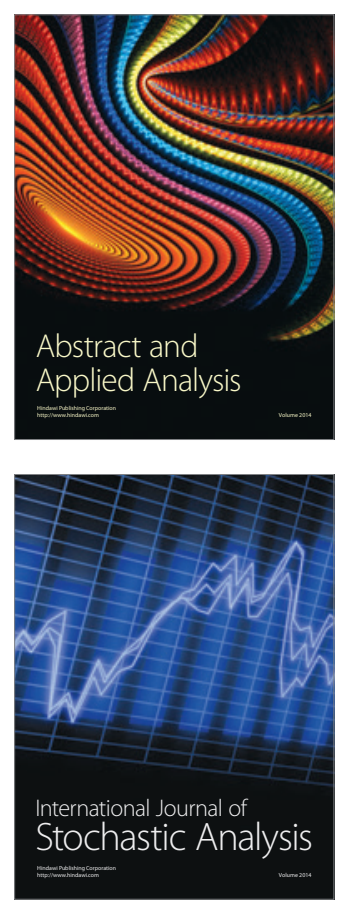

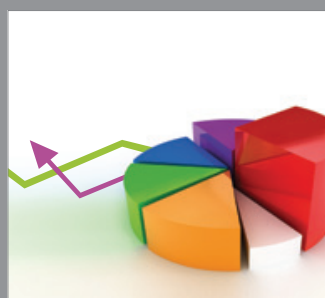

ournal of

Probability and Statistics

Promensencen
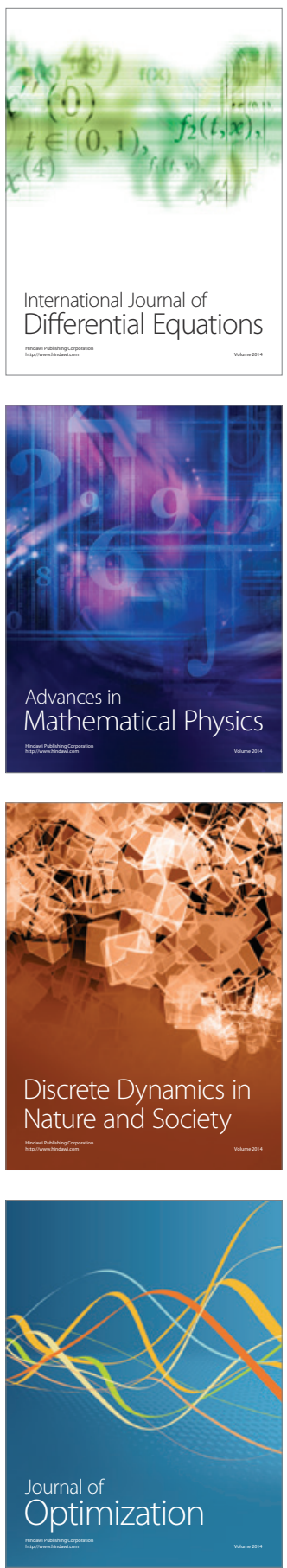